\title{
Conductivity between Luttinger liquids: Coupled chains and bilayer graphene
}

\author{
Vieri Mastropietro \\ Università di Roma Tor Vergata, Viale della Ricerca Scientifica, IT-00133 Roma, Italy \\ (Received 26 December 2010; revised manuscript received 31 March 2011; published 20 July 2011)
}

\begin{abstract}
The conductivity properties between Luttinger liquids are analyzed by exact renormalization-group methods. We prove that in a two chain system or in a model of bilayer graphene, described by two coupled Fermionic honeycomb lattices interacting with a gauge field, the transverse optical conductivity at finite temperature is anomalous and decreasing together with the frequency as a power law with a Luttinger liquid exponent.
\end{abstract}

DOI: 10.1103/PhysRevB.84.035109

PACS number(s): 05.10.Cc, 05.60.-k, 72.10.-d

\section{INTRODUCTION}

The many body interaction in Fermionic systems can destroy the electronlike nature of the elementary excitations, a fact which can have deep consequences on the transport properties. This was pointed out by Anderson, ${ }^{1}$ who got evidence that the coherent transport between Luttinger liquids due to particle hopping is strongly depressed with respect to the noninteracting case. One of the first applications of this idea was (see Refs. 2 and 3 ) an explanation of the $c$-axis anomalous conductivity between planes in high $T_{c}$ superconductors, with the assumption of Luttinger liquid behavior in the planes described by 2D square lattice Hubbard models; while intriguing, this theory suffers from the fact that no convincing theoretical evidence has been found up to now to substantiate such assumption. Subsequently, the attention was focused on one-dimensional systems, where Luttinger liquid behavior is surely present; in addition to a theoretical interest, the main physical motivation was that coupled Fermionic chains well describe quasi-one-dimensional organic conductors (see, e.g., Ref. 4). At zero temperature, renormalization-group or bosonization analysis apparently indicates that the hopping can destroy Luttinger liquid behavior in several regimes (see, e.g., Refs. 5-17). On the other hand at higher temperatures, the system still shows Luttinger liquid properties. ${ }^{18-21}$ The transverse conductivity between weakly coupled Fermionic chains at temperatures and frequencies greater than the hopping was shown to be $\sigma^{\perp}(\omega) \sim \omega^{\alpha}$ with $\alpha$ related to the Luttinger liquid exponent $\eta$; in particular, the value of the exponent $\alpha$ was claimed to be $\alpha=2 \eta$ in Ref. 22, where a tunneling approach was followed, and in Ref. 23, by using the Kubo formula; on the other hand, in Ref. 24, the different value $\alpha=2 \eta-1$ was found by using dynamical mean-field theory. The reason for these discrepancies relies on the fact that the computations were done in the so-called Tomonaga-Luttinger or g-ology approximation, in which the fermions close to the two Fermi points are described in terms of massless Dirac particles. This simplifies the computations and allows the use of powerful techniques like bosonization, but introduces spurious ultraviolet divergences in the conductivity which, therefore, needs a regularization, and different regularizations produce different results [see, e.g., the discussion in Ref. 24 after Eq. (3)].

In conclusion, there are at present no firm results on the conductivity between higher-dimensional Luttinger liquids, and even in one dimension, there are still ambiguities due to approximations and regularizations. In this paper, the conduc- tivity properties between Luttinger liquids at finite temperature are analyzed by the exact renormalization-group methods developed starting from Ref. 25; indeed, the suggestion of using such techniques for this problem dates back to Anderson himself, ${ }^{1}$ but their full development required a long time. In the case of coupled spinless chains, we get an exact expression for the transverse conductivity (the lattice furnishes the natural cutoff), which is, at temperatures and frequencies greater than the hopping,

$$
\sigma_{\beta}^{\perp}\left(\omega_{n}\right) \sim t^{2} \omega_{n}^{2 \eta-1},
$$

if $\omega_{n}=\frac{2 \pi}{\beta} n$. The analysis is based on the implementation of Ward identities in the renormalization group, with rigorous bounds for the corrections due to the lattice. ${ }^{26}$ Note also that the computation of the parallel conductivity gives in this regime $\sigma_{\beta}^{\|}\left(\omega_{n}\right) \sim \omega_{n}^{-1}$, that is, no anomalous exponent appears in the frequency dependence in that case.

In addition to justifying the anomalous exponent predicted by mean-field theory in Ref. 24 for the two chain problem, the exact renormalization-group methods can provide evidence for anomalous transverse conductivity between bidimensional Luttinger liquids, for which bosonization cannot be applied. Electrons on the honeycomb lattice interacting with a $U(1)$ gauge field, representing the retarded electromagnetic (em) interaction or the effect of disorder or ripples, ${ }^{27}$ have Luttinger liquid behavior. Indeed, this system was analyzed in the continuum Dirac approximation in the early work, ${ }^{28}$ where evidence of Luttinger liquid was found based on second-order perturbation theory. Later on, in Refs. 29 and 30, Luttinger liquid behavior was established at any order and, taking rigorously into account the honeycomb lattice, by implementing lattice Ward identities in the renormalization-group scheme. The behavior of the two point function is similar to the one of the spinless chain; the wave-function renormalization has a power law with exponent $\eta$. By coupling two interacting Fermionic honeycomb lattices by a hopping term, we get a model for bilayer graphene. ${ }^{31}$ The zero temperature properties of such a system are rather complex and still not completely understood (see, e.g., Refs. 32-34) and the review in Ref. 35. However, as in the case of coupled chains, at finite temperature and frequencies, the Luttinger liquid behavior of the uncoupled system can reveal itself by the transverse conductivity; in particular, we will show that, at temperatures and frequencies greater than the hopping,

$$
\sigma_{\beta}^{\perp}\left(\omega_{n}\right) \sim t^{2} \omega_{n}^{2 \eta}
$$


while $\sigma_{\beta}^{\perp}\left(\omega_{n}\right)$ is essentially constant in the noninteracting case. This confirms in two dimensions the Anderson idea: coherent transport between Luttinger liquids is depressed with respect to noninteracting systems or Fermi liquids. Moreover, the presence of anomalous Luttinger liquid exponents in the frequency dependence of the transverse conductivity of bilayer graphene could be revealed in future experiments, and is apparently consistent with the suppression of the $c$-axis conductivity in graphite. $^{36}$

The paper is organized in the following way. In Sec. II, we introduce the two chain model, described in terms of two one-dimensional spinless Fermionic systems with a quartic short ranged interaction and coupled by a hopping term. The model is analyzed through an exact renormalizationgroup analysis which is valid at temperatures greater than the renormalized interchain hopping; indeed, the finite temperature condition ensures that several effective interactions produced by the hopping, which should be relevant in the renormalization-group sense at zero temperature, are indeed irrelevant in this regime. The Fermionic propagator acquires an anomalous dimension (and so several other Fermionic bilinears), and this produces the power law behavior in the transverse conductivity; on the contrary, no anomalous dimension appears in the parallel conductivity due to a cancellations following from a Ward identity. In Sec. III, we consider a model of bilayer graphene, described in terms of fermions on the honeycomb lattice with a planar long range interaction [mediated by a $U(1)$ gauge field] and coupled via a hopping term. Again an exact renormalization-group analysis somewhat similar to the previous one, based on lattice Ward identities and valid at finite temperatures, allows us to show the presence of anomalous dimensions and the power law behavior in the conductivity. Finally, in the Appendix, a short computation of the conductivity in the noninteracting case is presented.

\section{THE TWO CHAIN MODEL}

We consider a two chain model described as two onedimensional interacting spinless Fermionic systems coupled by a hopping term; the Hamiltonian is

$$
H=H_{1}+H_{2}+P,
$$

where for $i=1,2, H_{i}=H_{i}^{(0)}+V_{i}$,

$$
\begin{aligned}
& H_{i}^{(0)}=-\frac{1}{2} \sum_{x=1}^{L-1}\left(a_{x+1, i}^{+} a_{x, i}^{-}+a_{x, i}^{+} a_{x+1, i}^{-}\right), \\
& V_{i}=-\lambda \sum_{x, y=1}^{L-1} v(x-y) a_{x, i}^{+} a_{x, i}^{-} a_{y, i}^{+} a_{y, i}^{-},
\end{aligned}
$$

and

$$
P=-t \sum_{x=1}^{L-1}\left[a_{x, 1}^{+} a_{x, 2}^{-}+a_{x, 2}^{+} a_{x, 1}^{-}\right]
$$

where $a_{x, i}^{ \pm}$are Fermionic operators and $|v(x)| \leqslant e^{-\kappa|x|}$. Either $\lambda$ and $t$ are assumed to be small.
As usual, we can introduce the interaction with an em field with a Peierls substitution $H \rightarrow H(A)$ with $V_{i}(A)=V$ and, if $A=\left(A^{\|}, A^{\perp}\right)$,

$$
H_{i}^{(0)}(A)=-\sum_{x} \frac{1}{2}\left(a_{x+1, i}^{+} e^{i A_{x}^{\|}} a_{x, i}^{-}+a_{x, i}^{+} e^{-i A_{x}^{\|}} a_{x+1, i}^{-}\right)
$$

and

$$
P(A)=-t \sum_{x}\left(a_{x, 1}^{+} e^{i A_{x}^{\perp}} a_{x, 2}^{-}+a_{x, 2}^{+} e^{-i A_{x}^{\perp}} a_{x, 1}^{-}\right) .
$$

The parallel current is defined as

$$
\frac{\partial H(A)}{\partial A_{x}^{\|}}=j_{x}^{P, \|}+A_{x}^{\|} j_{x}^{D, \|}+O\left(\left(A^{\|}\right)^{2}\right),
$$

where $j_{x, i}^{P, \|}$ and $j_{x, i}^{D, \|}$ are called, respectively, the paramagnetic and diamagnetic parts of the current and are given by

$$
\begin{aligned}
& j_{x}^{P, \|}=\sum_{i=1}^{2} \frac{1}{2 i}\left(a_{x+1, i}^{+} a_{x, i}^{-}-a_{x, i}^{+} a_{x+1, i}^{-}\right), \\
& j_{x}^{D, \|}=\sum_{i=1}^{2} \frac{1}{2}\left(a_{x+1, i}^{+} a_{x, i}^{-}+a_{x, i}^{+} a_{x+1, i}^{-}\right) .
\end{aligned}
$$

The transverse current is defined as

$$
\frac{\partial H(A)}{\partial A_{x}^{\perp}}=j_{x}^{P, \perp}+A_{x}^{\perp} j_{x, i}^{D, \perp}+O\left(\left(A^{\perp}\right)^{2}\right),
$$

where $j_{x}^{P, \perp}$ and $j_{x}^{D, \perp}$ are called, respectively, the paramagnetic and diamagnetic parts of the current and are given by

$$
\begin{aligned}
j_{x}^{P, \perp} & =\frac{t}{i}\left(a_{x, 1}^{+} a_{x, 2}^{-}-a_{x, 2}^{+} a_{x, 1}^{-}\right), \\
j_{x}^{D, \perp} & =t\left(a_{x, 1}^{+} a_{x, 2}^{-}+a_{x, 2}^{+} a_{x, 1}^{-}\right) .
\end{aligned}
$$

Finally, the Fermionic density is $\rho_{x}=a_{1, x}^{+} a_{1, x}+a_{2, x}^{+} a_{2, x}$.

If $\mathbf{p}=\left(\omega_{n}, p\right), \omega_{n}=\frac{2 \pi}{\beta} n, p=\frac{2 \pi}{L} m$, the transverse conductivity at finite temperature is given by

$$
\sigma_{\beta}^{\perp}\left(\omega_{n}\right)=\frac{1}{\omega_{n}} \lim _{p \rightarrow 0}\left[-\left\langle\hat{j}_{\mathbf{p}}^{P, \perp} ; \hat{j}_{-\mathbf{p}}^{P, \perp}\right\rangle+\left\langle j_{x}^{D, \perp}\right\rangle\right],
$$

where if $A=O_{\mathbf{x}_{1}} \cdots O_{\mathbf{x}_{n}},\langle A\rangle=\left.\frac{\operatorname{Tr}\left[e^{-\beta H} \mathbf{T}(A)\right]}{\operatorname{Tr}\left[e^{-\beta H}\right]}\right|_{T}$, with $\mathbf{T}$ being the time order product and $T$ denoting truncation. An analogous definition holds for the parallel conductivity.

In order to compute the conductivity (12), it is convenient to introduce a Grassmann integral representation for the correlation; we introduce the following generating functional

$$
e^{\mathcal{W}_{t, \lambda}\left(A^{\perp}\right)}=\int P(d \psi) e^{-\mathcal{V}(\psi)-B\left(A^{\perp}, \psi\right)},
$$

where $\psi_{\mathbf{x}, i}^{ \pm}$are Grassmann variables, $\mathbf{x}=\left(x_{0}, x\right), P(d \psi)$ is the Grassmann integration with propagator $\delta_{i, j} \hat{g}(\mathbf{k})$,

$$
\hat{g}(\mathbf{k})=\frac{1}{-i k_{0}+\cos k-\cos p_{F}},
$$


with $k_{0}=\frac{2 \pi}{\beta}\left(n+\frac{1}{2}\right), \mathcal{V}$ is the interaction given by, if $\int d \mathbf{x}=$ $\int_{-\beta / 2}^{\beta / 2} d x_{0} \sum_{x}$,

$$
\begin{aligned}
\mathcal{V}(\psi)= & -\lambda \sum_{i=1}^{2} \int d \mathbf{x} d \mathbf{y} v(\mathbf{x}-\mathbf{y}) \psi_{\mathbf{x}, i}^{+} \psi_{\mathbf{x}, i} \psi_{\mathbf{y}, i}^{+} \psi_{\mathbf{y}, i} \\
& -v \sum_{i=1}^{2} \int d \mathbf{x} \psi_{\mathbf{x}, i}^{+} \psi_{\mathbf{x}, i}-t \int d \mathbf{x}\left(\psi_{\mathbf{x}, 1}^{+} \psi_{\mathbf{x}, 2}^{-}+\psi_{\mathbf{x}, 2}^{+} \psi_{\mathbf{x}, 1}^{-}\right),
\end{aligned}
$$

with $v(\mathbf{x}-\mathbf{y})=\delta\left(x_{0}-y_{0}\right) v(x-y)$, and $v$ is a counterterm which is introduced to take into account the renormalization of the chemical potential; moreover,

$$
B\left(\psi, A^{\perp}\right)=-i \int d \mathbf{x} A_{\mathbf{x}}^{\perp}\left[\psi_{\mathbf{x}, 1}^{+} \psi_{\mathbf{x}, 2}^{-}-\psi_{\mathbf{x}, 2}^{+} \psi_{\mathbf{x}, 1}^{-}\right] .
$$

Defining $H_{t}(\mathbf{x})$ the Fourier transform of $\left\langle\hat{j}_{\mathbf{p}}^{P, \perp} ; \hat{j}_{-\mathbf{p}}^{P, \perp}\right\rangle$, we can write

$$
H_{t}(\mathbf{x})=\left.t^{2} \frac{\partial^{2}}{\partial A_{\mathbf{x}}^{\perp} \partial A_{\mathbf{0}}^{\perp}} \mathcal{W}_{t, \lambda}\left(A^{\perp}\right)\right|_{0} .
$$

The analysis of the functional integral (13) will be done by renormalization-group (RG) methods, integrating smaller and smaller momentum scales. The hopping $t$ introduces an intrinsic scale in the RG analysis. For scales greater than the (renormalized) hopping, it is natural to treat the hopping as a perturbation using the chain variables $\psi^{ \pm}$; on the other hand, at smaller scales the hopping cannot be considered a perturbation, and it is convenient to use the variables

$$
\hat{b}_{\mathbf{k}, 1}=\frac{1}{\sqrt{2}}\left[\hat{\psi}_{\mathbf{k}, 1}+\hat{\psi}_{\mathbf{k}, 2}\right], \quad \hat{b}_{\mathbf{k}, 2}=\frac{1}{\sqrt{2}}\left[\hat{\psi}_{\mathbf{k}, 1}-\hat{\psi}_{\mathbf{k}, 2}\right],
$$

in terms of which the free action is diagonal, but the $b_{\mathbf{k}, 1}^{ \pm}, b_{\mathbf{k}, 2}^{ \pm}$ have different Fermi momentum. Note that the temperature acts an infrared cutoff so that for temperatures not too small, only the first regime is present.

The first step of the RG analysis is the decomposition of the propagator $g(\mathbf{k})$ as a sum of propagators supported close to the two Fermi points $\pm p_{F}$ and more and more singular in the infrared region, labeled by a quasiparticle index $\alpha= \pm$ (labeling the Fermi points) and by an integer $h \leqslant 0$ :

$$
\hat{g}(\mathbf{k})=\hat{g}^{(1)}(\mathbf{k})+\sum_{h=h_{\beta}}^{0} \sum_{\alpha= \pm} \hat{g}_{\alpha}^{(h)}\left(\mathbf{k}-\mathbf{p}_{F}^{\alpha}\right),
$$

with $\mathbf{p}_{F}^{\alpha}=\left(0, p_{F}\right), \hat{g}_{\alpha}^{(h)}$ supported on $2^{h-1} \leqslant\left|\mathbf{k}-\mathbf{p}_{F}^{\alpha}\right| \leqslant 2^{h+1}$, and $g^{(1)}(\mathbf{k})$ having support far from the Fermi points. Note that $2^{h_{\beta}} \sim \pi / \beta$; the fact that the temperature is finite implies that there is a finite number of scales.

The RG integration procedure is defined recursively in the following way. Assume that we have integrated the fields $\psi_{i}^{(1)}, \psi_{i, \alpha}^{(0)}, \ldots, \psi_{i, \alpha}^{(h+1)}$; we get

$$
\begin{aligned}
e^{\mathcal{W}_{t, \lambda}\left(A^{\perp}\right)}= & e^{F_{h}\left(A^{\perp}\right)} \int P\left(d \psi^{(\leqslant h)}\right) \\
& \times \exp \left[-\mathcal{V}^{(h)}\left(\sqrt{Z_{h}} \psi^{(\leqslant h)}\right)-B^{(h)}\left(A^{\perp}, \sqrt{Z_{h}} \psi^{(\leqslant h)}\right)\right],
\end{aligned}
$$

where $P\left(d \psi^{(\leqslant h)}\right)$ is the Grassmanian quadratic integration with the propagator given by

$$
g_{\alpha}^{(\leqslant h)}(\mathbf{x})=\frac{1}{\beta L} \frac{1}{Z_{h}} \sum_{\mathbf{k}} e^{i \mathbf{k x}} \frac{\chi_{h}(\mathbf{k})}{-i k_{0}+\cos p_{F}-\cos \left(k+\alpha p_{F}\right)},
$$

where $\chi_{h}(\mathbf{k})$ is a smooth cutoff function with support $\mid \mathbf{k}-$ $\alpha \mathbf{p}_{F} \mid \leqslant 2^{h+1}$, and $Z_{h}$ is the wave-function renormalization. The single scale propagator $g_{\alpha}^{(h)}(\mathbf{x})$ is obtained from $\hat{g}_{\alpha}^{(\leqslant h)}(\mathbf{k})$, replacing $\chi_{h}(\mathbf{k})$ with $f_{h}(\mathbf{k})$ with support $2^{h-1} \leqslant\left|\mathbf{k}^{\prime}\right| \leqslant 2^{h+1}$, $\mathbf{k}=\left(k_{0}, k^{\prime}\right), k=k^{\prime}+\alpha p_{F}$, and $k^{\prime}$ is the momentum measured from the Fermi point. It can be written as

$$
g_{\alpha}^{(h)}(\mathbf{x})=e^{i \alpha p_{F} x} \frac{1}{\beta L} \frac{1}{Z_{h}} \sum_{\mathbf{k}} e^{i \mathbf{k}^{\prime} \mathbf{x}} \frac{f_{h}\left(\mathbf{k}^{\prime}\right)}{-i k_{0}+\alpha v_{F} k^{\prime}}+r^{(h)}(\mathbf{x}),
$$

with $v_{F}=\sin p_{F}, r^{(h)}(\mathbf{x})$ with the same decay properties as $g_{\alpha}^{(h)}(\mathbf{x})$ with an extra factor $2^{h}$; therefore, the more we are close to the Fermi momenta (i.e., $-h$ is large), the more $r^{(h)}(\mathbf{x})$ is a small correction and the propagator is essentially coinciding with the one of a massless Dirac particle. Finally, $\mathcal{V}^{(h)}$ is the effective potential expressed by a sum of monomials of order $n$ in the fields $\psi^{(\leqslant h)}$ multiplied times a kernel $W_{n, 0}^{(h)}$, while $B^{(h)}$ is sum of monomials of order $n$ in $\psi$ and $m$ in $A^{(\perp)}$ with kernels $W_{n, m}^{(h)}$. According to power counting, using that $\hat{g}_{\alpha}^{(h)}(\mathbf{k}) \sim 2^{-h}$ and $\int d \mathbf{k} \hat{g}_{\alpha}^{(h)}(\mathbf{k}) \sim 2^{h}$, the "naive" scaling dimension of such monomials is

$$
D=2-n / 2-m \text {. }
$$

In the RG analysis, we have to decompose $\mathcal{V}^{(h)}$ (a similar decomposition must be done also for $B^{(h)}$ ) as

$$
\mathcal{V}^{(h)}=\mathcal{L} \mathcal{V}^{(h)}+\mathcal{R} \mathcal{V}^{(h)},
$$

with $\mathcal{R}=1-\mathcal{L} ; \mathcal{L} \mathcal{V}^{(h)}$ is the relevant or marginal part of the effective interaction while $\mathcal{R} \mathcal{V}^{(h)}$ is the irrelevant part. Generally, this decomposition is dictated by the naive scaling dimension (22); $\mathcal{L}$ should select the terms with positive or vanishing dimension $D$. However, if the temperature verifies the condition

$$
2^{h_{\beta}}>t_{h_{\beta}}
$$

where $t_{h}$ is the hopping at scale $h$, there is an improvement with respect to naive power counting, and certain terms which are dimensionally relevant or marginal are indeed irrelevant. In order to verify this fact, we can split the kernels as $W_{n, m}^{(h)}=W_{n, m}^{(a)(h)}+W_{n, m}^{(b)(h)}$, where $W_{n, m}^{(a)(h)}$ is obtained from $W_{n, m}^{(h)}$ by setting $t=0$. In the case, $n=4, m=0$ (with vanishing scaling dimension),

$$
\mathcal{L} \hat{W}_{4,0}^{(h)}\left(\underline{\mathbf{k}}^{\prime}\right)=\hat{W}_{4,0}^{(a)(h)}(\underline{\mathbf{0}})
$$

So that

$$
\mathcal{R} \hat{W}_{4,0}^{(h)}\left(\underline{\mathbf{k}}^{\prime}\right)=\left[\hat{W}_{4,0}^{(a)(h)}\left(\mathbf{k}^{\prime}\right)-\hat{W}_{4,0}^{(a)(h)}(\underline{\mathbf{0}})\right]+\hat{W}_{4,0}^{(b)(h)}\left(\mathbf{k}^{\prime}\right) .
$$

The first term in the right-hand side (rhs) of (26) can be rewritten as $\underline{\mathbf{k}}^{\prime} \cdot \underline{\partial} W_{n, m}^{(a)(h)}$, and this produces an improvement $\sim 2^{h^{\prime}-h}$ in the bound of the kernel, if $h^{\prime}$ is the scale of the momentum, which is sufficient to make it irrelevant. Similarly 
the second term in (26), namely $\hat{W}_{4,0}^{(b)(h)}\left(\hat{\mathbf{k}}^{\prime}\right)$, has an extra $t_{h} 2^{-h} \leqslant 2^{\left(h_{\beta}-h\right)(1-\eta)}, \eta=O\left(\lambda^{2}\right)$ with respect to the bound for $W_{4,0}^{(h)}$, which again is enough to make it irrelevant; therefore, the true marginal contribution is given by the rhs of (25). Therefore, the only marginal quartic terms involve fermions with the same chain index, and the corresponding effective coupling coincides with the one of the uncoupled $t=0$ case.

Similarly, we define, for the terms with $n=2$ and the same chain index,

$$
\mathcal{L} \hat{W}_{2,0}^{(h)}\left(\underline{\mathbf{k}}^{\prime}\right)=\hat{W}_{2,0}^{(a)(h)}(\underline{\mathbf{0}})+\mathbf{k}^{\prime} \partial \hat{W}_{2,0}^{(a)(h)}(\underline{\mathbf{0}}) .
$$

Note that $W_{2,0}^{(b)(h)}$ has an extra $\left(t_{h} 2^{-h}\right)^{2}$ (there are no terms linear in $t_{h}$ by conservation of the chain index). Finally, if $n=2, m=0$ and the Fermionic fields have different chain index, then

$$
\mathcal{L} \hat{W}_{2,0}^{(h)}\left(\underline{\mathbf{k}}^{\prime}\right)=\hat{W}_{2,0}^{(h)}(\underline{\mathbf{0}}) .
$$

Note that the terms with $n=2$ and an extra derivative are irrelevant as they have at least an extra $t_{h} 2^{-h}$. Therefore,

$$
\begin{aligned}
\mathcal{L V}^{(h)}(\psi)= & \sum_{i=1}^{2}\left[\lambda _ { h } \int d \mathbf { x } \left(\psi_{\mathbf{x}, i,+}^{+} \psi_{\mathbf{x}, i,+}^{-} \psi_{\mathbf{x}, i,-}^{+} \psi_{\mathbf{x}, i,-}^{-}\right.\right. \\
& \left.+2^{h} \sum_{\alpha= \pm} v_{h} \psi_{\mathbf{x}, i, \alpha}^{+} \psi_{\mathbf{x}, i, \alpha}^{-}+\delta_{h} \sum_{\alpha= \pm} \psi_{\mathbf{x}, i, \alpha}^{+} \alpha v_{F} \partial_{x} \psi_{\mathbf{x}, i, \alpha}^{-}\right) \\
& \left.+t_{h} \sum_{\alpha= \pm} \int d \mathbf{x}\left(\psi_{\mathbf{x}, 1, \alpha}^{+} \psi_{\mathbf{x}, 2, \alpha}^{-}+\psi_{\mathbf{x}, 2, \alpha}^{+} \psi_{\mathbf{x}, 1, \alpha}^{-}\right)\right]
\end{aligned}
$$

In the above expression, $\lambda_{h}$ represents the effective interaction at momentum scale $h, \delta_{h}$ the effective Fermi velocity, $v_{h}$ the shift of the chemical potential, and $t_{h}$ the effective hopping. By definition, $Z_{h}, \lambda_{h}, v_{h}, \delta_{h}$ are exactly the same as in the theory with $t=0$. It is possible to choose $v$ so that $v_{h}$ remain small for any $h$. By combining Ward identities at each renormalizationgroup iteration together with the Schwinger-Dyson equation, it follows (see Ref. 26) that

$$
\lambda_{h} \rightarrow_{h \rightarrow-\infty} \lambda_{-\infty}(\lambda), \quad \delta_{h} \rightarrow_{h \rightarrow-\infty} \delta_{-\infty}(\lambda),
$$

with $\lambda_{-\infty}(\lambda), \delta_{-\infty}(\lambda)$ analytic functions of $\lambda$; moreover,

$$
Z_{h} \sim 2^{-\eta h},
$$

with $\eta$ analytic in $\lambda$ and $\eta=a \lambda^{2}+O\left(\lambda^{3}\right)$ with $a>0$. Moreover, in Ref. 26 (and references therein), it is also proven that kernels $W_{n, m}^{(h)}$ are analytic functions of $\left\{\lambda_{k}, v_{k}, \delta_{k}, t_{k}\right\}_{k \geqslant h}$ : analyticity (implying the "nonperturbative" nature of the method) is a very nontrivial property obtained exploiting anticommutativity properties of Grassmann variables, via Gram inequality for determinants and Bridges-Battle-Federbush formula for truncated expectations.

Regarding the flow of $t_{h}$, we obtain

$$
t_{h-1}=\frac{Z_{h}}{Z_{h-1}}\left(t_{h}+\beta_{t}^{(h)}\right),
$$

with $\left|\beta_{t}^{(h)}\right| \leqslant C_{1} t_{h} \lambda^{2}\left(t_{h} 2^{-h}\right)^{2}$. It is easy to see by induction that $\left|Z_{h} t_{h}-t\right| \leqslant C_{2} t|\lambda|$. Assume, indeed, that it is true for $k \geqslant h$; therefore, for $\lambda, t$ small enough,

$$
\left|t_{h-1} Z_{h-1}-t\right| \leqslant 2 t C_{1} \lambda^{2} \sum_{k=h}^{0}\left(t_{k} 2^{-k}\right)^{2},
$$

from which using (24), the inductive assumption follows. Note that the effective hopping, even if relevant in the RG sense according to naive power counting, remains small in this region of temperatures. Moreover, from (24) we obtain the condition between the temperature and the hopping:

$$
\beta^{-1} \geqslant t^{\frac{1}{1-\eta}}\left[1+O\left(\lambda^{2}\right)\right] .
$$

Regarding the effective source $B^{(h)}$, we define

$$
\mathcal{L} W_{2,1}^{(h)}\left(\mathbf{k}^{\prime}, \mathbf{p}\right)=W_{2,1}^{(a)(h)}(\mathbf{0 , 0})=1 .
$$

Indeed, the graphs contributing to $W_{2,1}^{(a)(h)}(\mathbf{0})$ are one particle reducible (as the interaction involves only fermions from the same chain), and $\left.g^{(h)}\left(\mathbf{k}^{\prime}\right)\right|_{\mathbf{k}^{\prime}=0}=0$. Therefore (assuming that $A_{\mathbf{p}}^{\perp}$ has small support around $\mathbf{p}=0$ ),

$$
\begin{aligned}
\mathcal{L} & B^{(h)}\left(A^{\perp}, \sqrt{Z_{h}} \psi\right) \\
& =-i \sum_{i=1}^{2} \sum_{\alpha= \pm} \int d \mathbf{x} A_{\mathbf{x}}^{\perp}\left(\psi_{\mathbf{x}, 1, \alpha}^{+} \psi_{\mathbf{x}, 2, \alpha}^{-}+\psi_{\mathbf{x}, 2, \alpha}^{+} \psi_{\mathbf{x}, 1, \alpha}^{-}\right) .
\end{aligned}
$$

As the flow of the effective parameters corresponding to the relevant and marginal operators is bounded, the following bound is obtained, for $\beta$ verifying (34):

$$
\frac{1}{L \beta} \int d \underline{\mathbf{x}}\left|W_{n, m}^{(h)}(\mathbf{x})\right| \leqslant C 2^{h\left(2-\frac{n}{2}-m\right)} .
$$

In order to compute the conductivity, we have to separate the terms proportional to $t^{2}$ in both the paramagnetic and diamagnetic contributions to (12) from the rest. We write the current-current correlation as

$$
t^{-2} H_{t}(\mathbf{x})=\left.\frac{\partial^{2} \mathcal{W}_{0, \lambda}}{\partial A_{\mathbf{x}}^{\perp} \partial A_{\mathbf{0}}^{\perp}}\right|_{0}+t^{-2} \tilde{H}_{t}(\mathbf{x}),
$$

where the first term in the rhs is independent of $t$ and, from (37) and $t \beta$ small,

$$
\int d \mathbf{x}\left|\tilde{H}_{t}(\mathbf{x})\right| \leqslant C t^{2} \sum_{h=h_{\beta}}^{0}\left(\frac{t}{2^{h}}\right)^{2} Z_{h}^{-4} \leqslant 2 t^{2} C\left(t \beta^{1-2 \eta}\right)^{2} .
$$

In order to compute the conductivity, we still have to compute $\left\langle j_{x}^{D, \perp}\right\rangle$; introducing the generating functional

$$
e^{\overline{\mathcal{W}}_{t, \lambda}(J)}=\int P(d \psi) \exp \left[-\mathcal{V}(\psi)-t \int d \mathbf{x} J_{\mathbf{x}} h_{\mathbf{x}}\right],
$$

where $h_{\mathbf{x}}=\psi_{\mathbf{x}, 1}^{+} \psi_{\mathbf{x}, 2}^{-}+\psi_{\mathbf{x}, 2}^{+} \psi_{\mathbf{x}, 1}^{-}$, we get

$$
\left\langle j_{x}^{D, \perp}\right\rangle=\left.\frac{\partial \overline{\mathcal{W}}_{t, \lambda}}{\partial J_{\mathbf{x}}}\right|_{0}=\left.t^{2} \int d \mathbf{x}_{1} \frac{\partial^{2} \overline{\mathcal{W}}_{0, \lambda}}{\partial J_{\mathbf{x}} \partial J_{\mathbf{x}_{1}}}\right|_{0}+\Delta,
$$

where

$$
\Delta=\left.\sum_{n=3}^{\infty} \frac{t^{n+1}}{n !} \int d \mathbf{x}_{1} \cdots \int d \mathbf{x}_{n} \frac{\partial^{n+1} \overline{\mathcal{W}}_{0, \lambda}}{\partial J_{\mathbf{x}} \partial J_{\mathbf{x}_{1}} \cdots \partial J_{\mathbf{x}_{n}}}\right|_{0}
$$


and only odd $n$ contribute. From the analog of (37), the left-hand side (lhs) is bounded by the sum over $h$ of $\sum_{n=3}^{\infty} t^{n+1} 2^{-h(n-1)} Z_{h}^{-4}$ so that for $t \beta$ small,

$$
|\Delta| \leqslant C_{1} t^{2} \sum_{h=h_{\beta}}^{0}\left(2^{-h} t Z_{h}^{-2}\right)^{2} \leqslant C_{2}^{n} t^{2}\left(t \beta^{1-2 \eta}\right)^{2} .
$$

Note finally that, if $\langle\ldots\rangle \equiv\langle\ldots\rangle_{t, \lambda}$

$$
\left\langle j_{\mathbf{x}}^{P, \perp} ; j_{\mathbf{y}}^{P, \perp}\right\rangle_{0, \lambda}=\left\langle j_{\mathbf{x}}^{D, \perp} ; j_{\mathbf{y}}^{D, \perp}\right\rangle_{0, \lambda},
$$

and this implies that there is an important cancellation between the paramagnetic and diamagnetic parts of the conductivity; indeed,

$$
\begin{aligned}
& -\left\langle j_{\mathbf{p}}^{P, \perp} ; j_{-\mathbf{p}}^{P, \perp}\right\rangle+\left\langle j_{x}^{D, \perp}\right\rangle \\
& =t^{2} \int d \mathbf{x}\left(e^{i \omega_{n} x_{0}}-1\right)\left\langle j_{\mathbf{x}}^{D, \perp} ; j_{\mathbf{y}}^{D, \perp}\right\rangle_{0, \lambda}+O\left(t^{2}\left(t \beta^{1-2 \eta}\right)^{2}\right),
\end{aligned}
$$

where

$$
\left\langle j_{\mathbf{x}}^{D, \perp} ; j_{\mathbf{y}}^{D, \perp}\right\rangle_{0, \lambda}=\left\langle\psi_{\mathbf{x}, 1}^{-} \psi_{\mathbf{y}, 1}^{+}\right\rangle_{0, \lambda}\left\langle\psi_{\mathbf{y}, 2}^{+} \psi_{\mathbf{x}, 2}^{-}\right\rangle_{0, \lambda} .
$$

Therefore, for $\omega_{n}$ small,

$$
\begin{aligned}
& \left|\int d \mathbf{x}\left(e^{i \omega_{n} x_{0}}-1\right)\left\langle j_{\mathbf{x}}^{D, \perp} ; j_{\mathbf{y}}^{D, \perp}\right\rangle_{0, \lambda}\right| \\
& \leqslant C_{1} \int_{|\mathbf{x}| \leqslant\left|\omega_{n}\right|^{-1}} d \mathbf{x} \frac{\left|x_{0} \omega_{n}\right|}{1+|\mathbf{x}|^{2+2 \eta}} \\
& \quad+C_{1} \int_{|\mathbf{x}| \geqslant\left|\omega_{n}\right|^{-1}} d \mathbf{x} \frac{1}{1+|\mathbf{x}|^{2+2 \eta}} \leqslant \frac{C_{2}}{\eta}\left|\omega_{n}\right|^{2 \eta} .
\end{aligned}
$$

In conclusion, the transverse conductivity is given by (1) for $t \ll \beta^{-1} \ll \omega_{n} \ll 1$. In the noninteracting case, $t^{-2} \omega_{n} \sigma_{\beta}^{\perp}\left(\omega_{n}\right) \sim \frac{2}{\pi \sin p_{F}}$ so that we can conclude that the presence of the interchain interaction decreases the transverse conductivity in this regime.

The transverse conductivity should be compared with the parallel conductivity $\sigma_{\beta}^{\|}$, defined as in (12), with $j^{D, \|}, j^{P, \|}$ replacing $j^{D, \perp}, j^{P, \perp}$. For $t \ll \beta^{-1} \ll \omega_{n} \ll 1$, one gets

$$
\omega_{n} \sigma_{\beta}^{\|}\left(\omega_{n}\right) \sim 2 \frac{v_{F} K}{\pi},
$$

where $K$ is the Luttinger liquid parameter $2 \eta=K+K^{-1}-2$. The above formula, which is part of the Haldane's Luttinger liquid conjecture, ${ }^{37}$ can be derived by a renormalization-group analysis analog like the one described above (see Ref. 38). The parallel current-current correlation is obtained by a generating functional similar to (13) in which $B\left(A^{\perp}, \psi\right)$ is replaced by $B\left(A^{\|}, \psi\right)=\int d \mathbf{x} A_{\mathbf{x}}^{\|} j_{\mathbf{x}}^{P, \|}$; after the integration of the fields $\psi_{i}^{(1)}, \psi_{i, \alpha}^{(0)}, \ldots, \psi_{i, \alpha}^{(h+1)}$, we get an expression similar to (19) with $B^{(h)}\left(A^{\perp}, \sqrt{Z_{h}} \psi\right)$ replaced by $B^{(h)}\left(A^{\|}, \sqrt{Z_{h}} \psi\right)$ with

$$
\mathcal{L} B^{(h)}\left(A^{\|}, \sqrt{Z_{h}} \psi\right)=Z_{h}^{(1)} \int d \mathbf{x} j_{\mathbf{x}}^{P, \|(\leqslant h)}
$$

and

$$
\frac{Z^{(1)}}{Z_{h}}=1+O(\lambda)
$$

as a consequence of a Ward identity. Therefore, the renormalization of the parallel current is proportional to the wavefunction renormalization [while there is no renormalization of the transverse current; see (35)], and this explains why anomalous power law exponents do not appear in the frequency dependence of the parallel conductivity. In conclusion, from (1) and (47), we see that $\eta$ can be independently determined in experiments on two chain systems either from the amplitude of the parallel conductivity or from the exponent in the orthogonal conductivity.

As a final remark, we stress that the above analysis is true only for temperatures greater than $\sim t^{\frac{1}{1-\eta}}$; at lowest temperature, the $\mathrm{RG}$ analysis would be identical to the previous one up to scale $t^{\frac{1}{1-\eta}}$, but at lowest scales, one should perform the change of variables (17); the system would be described in terms of two fermions with different Fermi momenta [the difference is $O\left(t^{\frac{1}{1-\eta}}\right)$ ]. In this second regime, the power counting improvement described above is not valid, and this produces several (not a single one, as in spinless Luttinger liquids) effective quartic couplings with a generically unbounded RG flow.

\section{BILAYER GRAPHENE}

An analysis similar to the previous one can be repeated for a model of bilayer graphene, described in terms of electrons on the honeycomb lattice interacting through a $U(1)$ quantized gauge field, which can represent either the em interaction or the effects of ripples or disorder (see, e.g., Ref. 27).

We introduce creation and annihilation Fermionic operators $\psi_{\vec{x}, i}^{ \pm}=\left(a_{\vec{x}, i}^{ \pm}, b_{\vec{x}+\vec{\delta}_{1}, i}^{ \pm}\right)=|\mathcal{B}|^{-1} \int_{\vec{k} \in \mathcal{B}} d \vec{k} \psi_{\vec{k}, i, \sigma}^{ \pm} e^{ \pm i \vec{k} \vec{x}}$ for electrons with plane indices $i=1,2$ and sitting at the sites of the two triangular sublattices $\Lambda_{A}$ and $\Lambda_{B}$ of a honeycomb lattice; we assume that $\Lambda_{A}$ has basis vectors $\vec{l}_{1,2}=\frac{1}{2}(3, \pm \sqrt{3})$ and that $\Lambda_{B}=\Lambda_{A}+\vec{\delta}_{j}$, with $\vec{\delta}_{1}=(1,0)$ and $\vec{\delta}_{2,3}=\frac{1}{2}(-1, \pm \sqrt{3})$ the nearest neighbor vectors; $\mathcal{B}$ is the first Brillouin zone and $|\mathcal{B}|=\frac{8 \pi^{2}}{3 \sqrt{3}}$. In the absence of em interaction, the Hamiltonian is

$$
H=H_{1}+H_{2}+P,
$$

where

$$
H_{i}=-\sum_{\substack{\vec{x} \in \Lambda_{A} \\ j=1,2,3}} a_{\vec{x}, i}^{+} b_{\vec{x}+\vec{\delta}_{j}, i}^{-}+\text {c.c. }
$$

describes the hopping of fermions in the plane and

$$
\begin{aligned}
P= & -t \sum_{\vec{x} \in \Lambda_{A}}\left[a_{\vec{x}, 1}^{+} a_{\vec{x}, 2}^{-}+a_{\vec{x}, 2}^{+} a_{\vec{x}, 1}^{-}\right. \\
& \left.+b_{\vec{x}+\vec{\delta}_{1}, 1}^{+} b_{\vec{x}+\vec{\delta}_{1}, 2}^{-}+b_{\vec{x}+\vec{\delta}_{1}, 2}^{+} b_{\vec{x}+\vec{\delta}_{1}, 1}^{-}\right]
\end{aligned}
$$

describes the Fermionic hopping from one plane to another; either $e$ and $t$ will be assumed small. The interaction with a transverse classical em field is introduced via the Peierls substitution. If $A^{\perp}$ is a classical external field,

$$
\begin{aligned}
P= & -t \sum_{\vec{x} \in \Lambda_{A}}\left[a_{\vec{x}, 1}^{+} e^{i A_{\vec{x}}^{\perp}} a_{\vec{x}, 2}^{-}+a_{\vec{x}, 2}^{+} e^{-i A_{\vec{x}}^{\perp}} a_{\vec{x}, 1}^{-}\right. \\
& \left.+b_{\vec{x}+\vec{\delta}_{1}, 1}^{+} e^{i A_{\vec{x}+\vec{\delta}_{1}}^{\perp}} b_{\vec{x}+\vec{\delta}_{1}, 2}^{-}+b_{\vec{x}+\vec{\delta}_{1}, 2}^{+} e^{-i A_{\vec{x}+\vec{\delta}_{1}}^{\perp}} b_{\vec{x}+\vec{\delta}_{1}, 1}^{-}\right],
\end{aligned}
$$


the paramagnetic and diamagnetic parts of the transverse current are

$$
\begin{aligned}
j_{\vec{x}}^{P, \perp}= & \left.\frac{\partial H(A)}{\partial A_{x}^{\perp}}\right|_{0}=\frac{t}{i}\left[a_{\vec{x}, 1}^{+} a_{\vec{x}, 2}^{-}+b_{\vec{x}+\vec{\delta}_{1}, 1}^{+} b_{\vec{x}+\vec{\delta}_{1}, 2}^{-}-a_{\vec{x}, 2}^{+} a_{\vec{x}, 1}^{-}\right. \\
& \left.-b_{\vec{x}+\vec{\delta}_{1}, 2}^{+} b_{\vec{x}+\vec{\delta}_{1}, 1}^{-}\right] \\
j_{\vec{x}}^{D, \perp}= & \frac{\partial^{2} H(A)}{\partial^{2} A_{x}^{\|}}=t\left[a_{\vec{x}, 1}^{+} a_{\vec{x}, 2}^{-}+b_{\vec{x}+\vec{\delta}_{1}, 1}^{+} b_{\vec{x}+\vec{\delta}_{1}, 2}^{-}+a_{\vec{x}, 2}^{+} a_{\vec{x}, 1}^{-}\right. \\
& \left.+b_{\vec{x}+\vec{\delta}_{1}, 2}^{+} b_{\vec{x}+\vec{\delta}_{1}, 1}^{-}\right]
\end{aligned}
$$

and the transverse conductivity is defined as in (12) divided by $\frac{3 \sqrt{3}}{2}$, the area of the hexagonal cell of the honeycomb lattice.

We assume now that the electrons interact through an $U(1)$ gauge field; the current-current correlation is obtained from the following generating functional

$$
e^{\mathcal{W}_{t, e}\left(A^{\perp}\right)}=\int P(d \psi) P(d A) e^{-\mathcal{V}(\psi, A)-B\left(A^{\perp}, \psi\right)},
$$

where $\psi=(a, b)$ a couple of Grassmann variables (with slight abuse of notation, the Grassmann and the Fermionic operators are denoted with the same symbol), and $P(d \psi)$ is the Fermionic Gaussian integration for $\psi_{\mathbf{k}, i}^{ \pm}(i=1,2$ denotes the plane), $\mathbf{k}=k_{0}, \vec{k}, k_{0}=\frac{2 \pi}{\beta}\left(n+\frac{1}{2}\right)$, with propagator $\delta_{i, j} g(\mathbf{k})$ with

$$
g^{-1}(\mathbf{k})=-\left(\begin{array}{cc}
i k_{0} & v_{0} \Omega^{*}(\vec{k}) \\
v_{0} \Omega(\vec{k}) & i k_{0}
\end{array}\right),
$$

where $v_{0}=\frac{3}{2}$ and $\Omega(\vec{k})=\frac{2}{3} \sum_{j=1}^{3} e^{i \vec{k}\left(\vec{\delta}_{j}-\vec{\delta}_{1}\right)}$. The complex dispersion relation $\Omega(\vec{k})$ vanishes only at the two Fermi points $\vec{p}_{F}^{ \pm}=\left(\frac{2 \pi}{3}, \pm \frac{2 \pi}{3 \sqrt{3}}\right)$ and close to them assumes the form of a relativistic dispersion relation $\Omega\left(\vec{p}_{F}^{ \pm}+\vec{k}^{\prime}\right) \simeq i k_{1}^{\prime} \pm k_{2}^{\prime}$. Moreover

$$
\begin{aligned}
\mathcal{V}= & -\int d \mathbf{x}\left\{a_{\mathbf{x}, i, \sigma}^{+} b_{\mathbf{x}+\delta_{j}, \sigma} \exp \left[i e \int_{0}^{1} \vec{\delta}_{j} \cdot \vec{A}_{i}\left(\mathbf{x}+s \delta_{j}\right) d s\right]\right. \\
& + \text { c.c. }\}+\int d \mathbf{x} A_{\mathbf{x}, i}^{(0)} a_{\mathbf{x}, i}^{+} a_{\mathbf{x}, i, \sigma}+A_{\mathbf{x}+\delta_{1}, i}^{(0)} b_{\mathbf{x}+\delta_{1}, i}^{+} b_{\mathbf{x}+\delta_{1}, i},
\end{aligned}
$$

where $\int d \mathbf{x} \equiv \sum_{x \in \Lambda_{A}} \int d x_{0}$ and $A_{\mu, i}=\left(\vec{A}_{i}, A_{i}^{(0)}\right)$ is a boson field with propagator $\delta_{i, j} w(\mathbf{p})$ with

$$
w(\mathbf{p})=\frac{\chi\left(\sqrt{\omega_{n}^{2}+c^{2} p^{2}}\right)}{\sqrt{\omega_{n}^{2}+c^{2} p^{2}}},
$$

where $\chi$ is a cutoff function forbidding momenta either too large or smaller than the temperature. Finally, the source term is given by

$$
B\left(A^{\perp}, \psi\right)=\int d \mathbf{x} A_{\mathbf{x}}^{\perp} j_{\mathbf{x}}^{P, \perp} .
$$

We proceed exactly as in the previous case, writing the photon propagator as a sum of propagators more and more singular in the infrared region, and the Fermionic propagator as a sum of propagators supported close to the two Fermi points $\vec{p}_{F}^{ \pm}=$ $\left(\frac{2 \pi}{3}, \pm \frac{2 \pi}{3 \sqrt{3}}\right)$, labeled by a quasiparticle index $\alpha= \pm$ (labeling the Fermi points) and by an integer $h \leqslant 0$ :

$$
\begin{gathered}
w(\mathbf{p})=\sum_{h=h_{\beta}}^{1} w^{(h)}(\mathbf{p}), \\
g(\mathbf{k})=g^{(1)}(\mathbf{k})+\sum_{h=h_{\beta}}^{0} \sum_{\alpha= \pm} g_{\alpha}^{(h)}\left(\mathbf{k}-\mathbf{p}_{F}^{\alpha}\right),
\end{gathered}
$$

with $w^{(h)}(\mathbf{p})$ supported in $2^{h-1} \leqslant|\mathbf{p}| \leqslant 2^{h+1}, g_{\alpha}^{(h)}$ supported on $2^{h-1} \leqslant\left|\mathbf{k}-\mathbf{p}_{F}^{\alpha}\right| \leqslant 2^{h+1}$, and $g^{(1)}(\mathbf{k})$ having support far from the Fermi points.

Assume that we have integrated out the fields $\psi^{(1)}, \ldots, A^{(h+1)}, \psi^{(h+1)}, h \geqslant h_{\beta}$ so that

$$
\begin{aligned}
e^{W\left(A^{\perp}\right)}= & e^{F_{h}\left(A^{\perp}\right)} \int P\left(d \psi^{(\leqslant h}\right) \int P\left(d A^{(\leqslant h)}\right) \\
& \times \exp \left[\mathcal{V}^{(h)}\left(A, \sqrt{Z_{h}} \psi\right)+B_{h}\left(A^{\perp}, \sqrt{Z_{h}} \psi\right)\right],
\end{aligned}
$$

where $P\left(d A^{(\leqslant h)}\right)$ is the gauge-field integration with propagator $\delta_{i, j} \delta_{\mu, \nu} w^{\leqslant h}(\mathbf{p})$, with $w^{\leqslant h}(\mathbf{p})=\sum_{k=-\infty}^{h} w^{(k)}(\mathbf{p})$, while $P\left(d \psi^{(\leqslant h}\right)$ is the integration of the Fermionic field $\psi_{i, \alpha}$ with propagator $\delta_{i, i^{\prime}} \delta_{\alpha, \alpha^{\prime}} g_{\alpha}^{\leqslant h}\left(\mathbf{k}-\mathbf{p}_{F}^{(\alpha)}\right)$ with, if $\mathbf{k}^{\prime}=\mathbf{k}-\mathbf{p}_{F}^{(\alpha)}$,

$$
\begin{aligned}
g_{\alpha}^{(\leqslant h)}\left(\mathbf{k}^{\prime}\right)= & \frac{\chi_{h}\left(\mathbf{k}^{\prime}\right)}{Z_{h}}\left(\begin{array}{cc}
-i k_{0} & v_{h}\left(i k_{1}^{\prime}-\alpha k_{2}^{\prime}\right) \\
v_{h}\left(-i k_{1}^{\prime}-\alpha k_{2}^{\prime}\right) & -i k_{0}
\end{array}\right)^{-1} \\
& \times\left(1+R_{\omega}^{(h)}\right) .
\end{aligned}
$$

In Eq. (62), $\chi_{h}\left(\mathbf{k}^{\prime}\right)$ is a cutoff function with support in $\left|\mathbf{k}^{\prime}\right| \leqslant 2^{h}$ and $\left|R_{\omega}^{(h)}\left(\mathbf{k}^{\prime}\right)\right| \leqslant C\left|\mathbf{k}^{\prime}\right|^{\theta}$ for some $\theta>0$, while $Z_{h}$ and $v_{h}$ are, respectively, the effective wave-function renormalization and Fermi velocity on scale $h$.

The effective potential $\mathcal{V}^{(h)}+B^{(h)}$ is expressed by a sum of monomials of order $n$ in the fields $\psi^{(\leqslant h)}, m$ in $A^{(\mu)(\leqslant h)}$ and $l$ in $A^{\perp}$, multiplied by kernels $W_{n, m, l}^{(h)}$. According to power counting, the naive scaling dimension of such monomials is

$$
D=3-n-m-l \text {. }
$$

Again there is a dimensional improvement with respect to power counting if we are in a range of temperatures larger than the hopping, that is $2^{h_{\beta}}>t_{h_{\beta}}$, where $t_{h}$ is the hopping at scale $h$. We can split the kernels as $W_{n, m, l}^{(h)}=W_{n, m, l}^{(a)(h)}+W_{n, m, l}^{(b)(h)}$ where $W_{n, m, l}^{(b)(h)}$ is obtained from $W_{n, m}^{(h)}$ setting $t=0$. We define the $\mathcal{L}$ operator in the following way:

$$
\mathcal{L} \hat{W}_{2,1,0}^{(h)}\left(\mathbf{k}^{\prime}\right)=\hat{W}_{2,1,0}^{(a)(h)}(0) .
$$

Note, indeed, that the extra $t_{h} 2^{-h} \leqslant 2^{\left(h_{\beta}-h\right)(1-\eta)}, \eta=O\left(e^{2}\right)$ in $W_{2,1,0}^{(b)(h)}(0)$ is sufficient to make it irrelevant. Regarding the terms quadratic in the gauge fields, $\mathcal{L} \hat{W}_{0,2,0}^{(h)}(\mathbf{p})=\hat{W}_{0,2,0}^{(a)(h)}(0)+$ p $\partial \hat{W}_{0,2,0}^{(a)(h)}(0)$, where we have used that $\hat{W}_{0,2,0}^{(b)(h)}(0)$ has an extra $\left(2^{-h} t_{h}\right)^{2}$ with respect to the naive dimension; moreover, either $\hat{W}_{0,2,0}^{(a)(h)}(0)$ or $\partial \hat{W}_{0,2,0}^{(a)(h)}(0)$ are vanishing as consequence of the gauge symmetry (see Ref. 30). Finally, the terms quadratic in the Fermionic variables, if they have the same plane index, then $\mathcal{L} \hat{W}_{2,0,0}^{(h)}\left(\mathbf{k}^{\prime}\right)=\hat{W}_{2,0,0}^{(a)(h)}(0)+\mathbf{k}^{\prime} \partial \hat{W}_{2,0,0}^{(a)(h)}\left(\mathbf{k}^{\prime}\right)$, where we have used that in $\hat{W}_{2,0,0}^{(b)(h)}$, there is an extra gain $O\left(\left(t_{h} 2^{-h}\right)^{2}\right)$, 
due to the conservation of the plane index $i$. On the other hand, for the quadratic terms with a different plane index,

$$
\mathcal{L} \hat{W}_{2,0,0}^{(h)}\left(\mathbf{k}^{\prime}\right)=\hat{W}_{2,0,0}^{(h)}(\mathbf{0}) .
$$

Therefore,

$$
\begin{aligned}
\mathcal{L} \mathcal{V}^{(h)}(A, \psi)= & t_{h} \int d \mathbf{x} j_{\mathbf{x}}^{D, \perp}+\sum_{\mu, i, \alpha} \bar{e}_{\mu, h} \int \frac{d \mathbf{k}}{(2 \pi)|\mathcal{B}|} \\
& \times \frac{d \mathbf{p}}{(2 \pi)^{3}} \psi_{\mathbf{k}+\mathbf{p}, i, \alpha}^{+} \Gamma_{\mu}^{\omega} \psi_{\mathbf{k}, i, \alpha}^{-} A_{i}^{\mu}(\mathbf{p}),
\end{aligned}
$$

where $\bar{e}_{0, h}=e_{0, h}, \bar{e}_{i, h}=v_{h} e_{i, h}, e_{1, h}=e_{2, h}$ (thanks to discrete rotational symmetry), $\Gamma_{\mu}^{\omega}:=\Gamma_{\mu}\left(\vec{p}_{F}^{\omega}, \overrightarrow{0}\right)$ (with $\Gamma_{0}^{\omega}=-i I$, $\Gamma_{1}^{\omega}=-\sigma_{2}, \Gamma_{2}^{\omega}=-\omega \sigma_{1}$, and $\sigma_{1,2}$ are the first two Pauli matrices), and $\mathcal{R} \mathcal{V}^{(h)}, \mathcal{R}=1-\mathcal{L}$ is a sum of terms that are irrelevant in the RG sense. By construction, the flow of the effective parameters is the same as in the model with $t=0$; it was shown in Ref. 30, by a rigorous implementation of Ward identities in the RG scheme that the effective charges flow to a line of fixed points and the Fermi velocity increases up to the light velocity:

$$
e_{h} \rightarrow e_{-\infty}, \quad v_{h} \rightarrow c
$$

Moreover, the wave-function renormalization $Z_{h}$ diverges with anomalous exponents

$$
Z_{h} \sim 2^{-\eta h}, \quad \eta=\frac{e^{2}}{12 \pi^{2}}+\cdots .
$$

Finally, regarding the flow of $t_{h}$, we obtain

$$
t_{h-1}=\frac{Z_{h}}{Z_{h-1}}\left(t_{h}+\beta_{t}^{(h)}\right)
$$

with $\left|\beta_{t}^{(h)}\right| \leqslant C_{1} e^{6} t_{h}\left[\frac{t_{h}}{2^{h}}\right]^{2}$, and again by induction $\mid Z_{h} t_{h}-$ $t \mid \leqslant C_{2} t e^{6}$. We assume that the temperature verifies (24) which implies $\beta^{-1} \geqslant t^{\frac{1}{1-\eta}}\left[1+O\left(e^{2}\right)\right]$. Regarding the effective source $B^{(h)}$, we define $\mathcal{L} W_{2,0,1}^{(h)}\left(\mathbf{k}^{\prime}, \mathbf{p}\right)=W_{2,0,1}^{(a)(h)}(\mathbf{0 , 0})=1$, as again the graphs contributing to $W_{2,0,1}^{(a)(h)}(\mathbf{0})$ are one particle reducible and $\left.g^{(k)}\left(\mathbf{k}^{\prime}\right)\right|_{\mathbf{k}^{\prime}=0}=0$. As the flow of the effective parameters corresponding to the relevant and marginal operators is bounded, the following bound is obtained for $h \geqslant h_{\beta}$ (order by order in the renormalized expansion):

$$
\frac{1}{\Lambda \beta} \int d \underline{\mathbf{x}}\left|W_{n, m, l}^{(h)}(\underline{\mathbf{x}})\right| \leqslant C 2^{h(3-n-m-l)} .
$$

Using the same notation as in (38),

$$
\int d \mathbf{x}\left|x_{0}\right|\left|\tilde{H}_{t}(\mathbf{x})\right| \leqslant C t^{2} \sum_{h=h_{\beta}}^{0}\left(\frac{t}{2^{h}}\right)^{2} Z_{h}^{-4} \leqslant 2 t^{2} C\left(t \beta^{1-2 \eta}\right)^{2} .
$$

Moreover, as in the previous case, we introduce a generating functional $\overline{\mathcal{W}}_{t, e}(J)$ with source $t \int d \mathbf{x} J_{\mathbf{x}} h_{\mathbf{x}}$, where $j_{\mathbf{x}}^{D, \perp}=t h_{\mathbf{x}}$; we get

$$
\left\langle j_{x}^{D, \perp}\right\rangle=\left.t^{2} \int d \mathbf{x}_{1} \frac{\partial^{2} \overline{\mathcal{W}}_{0, e}}{\partial J_{\mathbf{x}} \partial J_{\mathbf{x}_{1}}}\right|_{0}+\Delta .
$$

From the analog of (70), the lhs is bounded by the sum over $h$ of $\sum_{n=3}^{\infty} t^{n+1} 2^{-h(n-2)} Z_{h}^{-4}$ so that for $t \beta$ small,

$$
\omega_{n}^{-1}|\Delta| \leqslant t^{2} \sum_{h=h_{\beta}}^{0} \beta 2^{h}\left[\frac{t 2^{-h}}{Z_{h}^{2}}\right]^{2} \leqslant C t^{2}\left(t \beta^{1-2 \eta}\right)^{2} .
$$

Note finally that

$$
\left\langle j_{\mathbf{x}}^{P, \perp} ; j_{\mathbf{y}}^{P, \perp}\right\rangle_{0, e}=\left\langle j_{\mathbf{x}}^{D, \perp} ; j_{\mathbf{y}}^{D, \perp}\right\rangle_{0, e}
$$

and

$$
\begin{aligned}
& \omega_{n}^{-1}\left|\int d \mathbf{x}\left(e^{i \omega_{n} x_{0}}-1\right)\left\langle j_{\mathbf{x}}^{D, \perp} ; j_{\mathbf{y}}^{D, \perp}\right\rangle_{0, e}\right| \\
& \leqslant \frac{C}{\omega_{n}}\left[\int_{|\mathbf{x}| \leqslant \omega_{n}^{-1}} d \mathbf{x}\left|x_{0} \omega_{n}\right|+\int_{|\mathbf{x}| \geqslant \omega_{n}^{-1}} d \mathbf{x}\right] \frac{1}{1+|\mathbf{x}|^{4+2 \eta}} \\
& \leqslant \frac{C_{2}}{\eta}\left|\omega_{n}\right|^{2 \eta} .
\end{aligned}
$$

Therefore, the conductivity in the interacting case is given by (2) for $t \ll \beta^{-1} \ll \omega_{n} \ll 1$, that is, the transverse conductivity decreases with the frequency with the anomalous exponent $2 \eta$. In absence of planar interaction, $t^{-2} \sigma_{\beta}^{\perp}\left(\omega_{n}\right)$ is essentially constant in this region, so that we can conclude that the presence of planar long-range interaction producing Luttinger liquid behavior decreases the transverse conductivity. Note also that the parallel conductivity does not display any anomalous power law, as a consequence of a Ward identity implying the analog of (49) (see Ref. 30).

\section{ACKNOWLEDGMENT}

The author gratefully acknowledges financial support from the ERC Starting Grant No. CoMBoS-239694.

\section{APPENDIX: THE NONINTERACTING CASE}

In the case of the two chain model if $\lambda=0$,

$$
\begin{aligned}
t^{-2} \omega_{n} \sigma_{\beta}^{\perp}\left(\omega_{n}\right)= & {\left[\int d \mathbf{k} g(\mathbf{k}+\mathbf{p}) g(\mathbf{k})\right.} \\
& \left.-\int d \mathbf{k} g(\mathbf{k}) g(\mathbf{k})\right]\left.\right|_{p=0}+O\left((\beta t)^{2}\right) .
\end{aligned}
$$

Note that

$$
\left.\lim _{\beta, L \rightarrow \infty} \frac{1}{L \beta} \sum_{\mathbf{k}} g(\mathbf{k}+\mathbf{p}) g(\mathbf{k})\right|_{p=0}=0,
$$

while

$$
\lim _{\beta, L \rightarrow \infty} \frac{1}{L \beta} \sum_{\mathbf{k}} g(\mathbf{k}) g(\mathbf{k})=-\frac{2}{\pi \sin p_{F}} .
$$

In the case of bilayer graphene, we get

$$
\begin{aligned}
t^{-2} \sigma_{\beta}^{\perp}\left(\omega_{n}\right)= & \frac{2}{3 \sqrt{3}} \frac{1}{\omega_{n}} \int \frac{d k_{0}}{2 \pi} \int \frac{d \vec{k}}{|\beta|}[F(\mathbf{k}, \mathbf{k}+\mathbf{p}) \\
& -F(\mathbf{k}, \mathbf{k})]\left.\right|_{\vec{p}=0}+O\left((t \beta)^{2}\right),
\end{aligned}
$$


where

$$
\begin{aligned}
F\left(\mathbf{k}_{1}, \mathbf{k}_{2}\right)= & 2\left[g_{11}\left(\mathbf{k}_{1}\right) g_{11}\left(\mathbf{k}_{2}\right)+g_{22}\left(\mathbf{k}_{1}\right) g_{22}\left(\mathbf{k}_{2}\right)\right. \\
& \left.+g_{12}\left(\mathbf{k}_{1}\right) g_{12}\left(\mathbf{k}_{2}\right)+g_{21}\left(\mathbf{k}_{1}\right) g_{21}\left(\mathbf{k}_{2}\right)\right] .
\end{aligned}
$$

The first term in the rhs can be written as its value for $\beta=\infty$ plus the rest $O\left(\beta^{-1}\right)$; the integral in the limit $\beta=\infty$ can be decomposed in a part integrated in the region $|\Omega(\vec{k})| \leqslant \varepsilon$ and $|\Omega(\vec{k})| \geqslant \varepsilon$; the second term is vanishing for $\omega_{n}=0$, while in the first, the contribution from the first two terms in (A3) are vanishing by parity, while the rest gives a nonzero constant at the vanishing external frequency.
${ }^{1}$ P. W. Anderson, Phys. Rev. Lett. 67, 3844 (1991).

${ }^{2}$ P. W. Anderson, Science 268, 1154 (1995).

${ }^{3}$ D. G. Clarke, S. P. Strong, and P. W. Anderson, Phys. Rev. Lett. 74, 4499 (1995).

${ }^{4}$ T. Giamarchi, Chem. Rev. 104, 5037 (2004)

${ }^{5}$ C. Bourbonnais and L. G. Caron, Int. J. Mod. Phys. B 5, 1033 (1991).

${ }^{6}$ V. M. Yakovenko, JETP Lett. 56, 523 (1992).

${ }^{7}$ A. Nersesyan, A. Luther, and F. Kusmartsev, Phys. Lett. A 176, 363 (1993).

${ }^{8}$ M. Fabrizio, Phys. Rev. B 48, 15838 (1993).

${ }^{9}$ A. M. Finkelstein and A. I. Larkin, Phys. Rev. B 47, 10461 (1993).

${ }^{10}$ L. Balents and M. P. A. Fisher, Phys. Rev. B 53, 12133 (1996).

${ }^{11}$ H. J. Schulz, Phys. Rev. B 53, R2959 (1996).

${ }^{12}$ E. Arrigoni, Phys. Rev. Lett. 80, 790 (1998).

${ }^{13}$ U. Ledermann and K. Le Hur, Phys. Rev. B 61, 2497 (2000).

${ }^{14}$ K. LeHur, Phys. Rev. B 63, 165110 (2001).

${ }^{15}$ P. Donohue, M. Tsuchiizu, T. Giamarchi, and Y. Suzumura, Phys. Rev. B 63, 045121 (2001).

${ }^{16}$ S. Dusuel and B. Doucot, Phys. Rev. B 67, 205111 (2003).

${ }^{17}$ S. Ledowski, P. Kopietz, and A. Ferraz, Phys. Rev. B 71, 235106 (2005).

${ }^{18}$ C. Bourbonnais and L. C. Caron, J. Phys. Lett. 45, L55 (1984).

${ }^{19}$ X. G. Wen, Phys. Rev. B 42, 6623 (1990).

${ }^{20}$ D. Boies, C. Bourbonnais, and A.-M. S. Tremblay, Phys. Rev. Lett. 74, 968 (1995).

${ }^{21}$ S. Capponi, D. Poilblanc, and F. Mila, Phys. Rev. B 54, 17547 (1996)
${ }^{22}$ D. G. Clarke, S. P. Strong, and P. W. Anderson, Phys. Rev. Lett. 72 , 3218 (1994).

${ }^{23}$ J. Moser et al., Eur. Phys. J. B 1, 39 (1998).

${ }^{24}$ A. Georges, T. Giamarchi, and N. Sandler, Phys. Rev. B 61, 16393 (2000).

${ }^{25}$ G. Benfatto and G. Gallavotti, Phys. Rev. B 42, 9967 (1990).

${ }^{26} \mathrm{G}$. Benfatto and V. Mastropietro, Commun. Math. Phys. 258, 609 (2005).

${ }^{27}$ M. A. H. Vozmediano, M. I. Katsnelson, and F. Guinea, Phys. Rep. 496, 109 (2010).

${ }^{28}$ J. Gonzalez, F. Guinea, and M. A. H. Vozmediano, Nucl. Phys. B 424, 595 (1994)

${ }^{29}$ A. Giuliani, V. Mastropietro, and M. Porta, Annales H. Poincare' 11, 1409 (2010).

${ }^{30}$ A. Giuliani, V. Mastropietro, and M. Porta, Phys. Rev. B 82, 121418 (2010).

${ }^{31}$ E. McCann and V. I. Fal'ko, Phys. Rev. Lett. 96, 086805 (2006).

${ }^{32}$ E. V. Castro, K. S. Novoselov, S. V. Morozov, N. M. R. Peres, J. M. B. Lopes dos Santos, J. Nilsson, F. Guinea, A. K. Geim, and A. H. C. Neto, Phys. Rev. Lett. 99, 216802 (2007).

${ }^{33}$ Y. Barlas and K. Yang, Phys. Rev. B 80, 161408(R) (2009).

${ }^{34}$ R. Nandkishore and L. Levitov, Phys. Rev. B 82, 115431 (2010).

${ }^{35}$ V. N. Kotov, B. Uchoa, V. M. Pereira, A. H. Castro Neto, and F. Guinea, e-print arXiv:1012.3484 (submitted to Rev. Mod. Phys.).

${ }^{36}$ L. Edman, B. Sundqvist, E. McRae, and E. Litvin-Staszewska, Phys. Rev. B 57, 6227 (1998).

${ }^{37}$ F. D. M. Haldane, Phys. Rev. Lett. 45, 1358 (1980).

${ }^{38}$ G. Benfatto and V. Mastropietro, J. Stat. Phys. 138, 1084 (2010). 